\title{
Examining and Comparing Executive Functions in Patients with Type 2 Diabetes Subjects and Healthy Subjects
}

\author{
Ahmad Alipour ${ }^{1}$ \\ Fariborz Bagheri² \\ Mahnaz AliAkbari-Dehkordi ${ }^{3}$ \\ Fatemeh Amirabadi ${ }^{*}$ \\ ${ }^{1}$ Department of Psychology, Faculty of Psychology, Payam-e-Noor University, Tehran, Iran \\ 2Department of Psychology, Faculty of Psychology, Science \& Research Branch, Islamic Azad University, Tehran, Iran \\ ${ }^{3}$ Department of Psychology, Faculty of Psychology, Payam-e-Noor University, Tehran, Iran \\ ${ }^{4}$ Department of Psychology, Faculty of Psychology, Payam-e-Noor University, Tehran, Iran \\ ${ }^{*}$ Corresponding Author
}

Doi:10.5901/mjss.2017.v8n3p293

\begin{abstract}
Today, diabetes is a serious problem worldwide, as WHO predicts, in the year 2025, the population suffering from diabetes will be about 333 million adults (Norouzi, Ghafranipour, Heidarnia, Tahmasbi, 2010). Disease Management requires planning and problem solving capabilities which as a range of functions are categorized as executive functions. Executive functions are important structures playing a fundamental role in directing and normalizing behavior. Therefore, the study was designed with the goal of comparing and examining patients suffering from type 2 diabetes in contrast with healthy subjects. The study was conducted using a causative-comparative method in 2013-14 in Tehran. Research population were people referring to Gabric association of whom 36 volunteer patients with diabetes were chosen. The control group were chosen amongst patients' companions with no disease records and were tested by WCST. Findings showed deficiencies in executive functions of patients with type 2 diabetes. Generally, we can say that these deficiencies can prevent proper disease management of type 2 diabetes.
\end{abstract}

Keywords: diabetes, executive functions, WCST.

\section{Introduction}

Today, diabetes is a serious problem worldwide, as WHO predicts, in the year 2025, the population suffering from diabetes will be about 333 million adults (Norouzi et al, 2010). On the other hand, WHO estimates diabetes patients to be more than 6 million people by 2030 (WHO, 2006). With regards to diabetes having several impacts, the disease imposes heavy costs on the community. Thus, identifying factors assisting patients manage their disease can help them reduce the costs. Disease management requires planning and problem solving capabilities, which as a range of functions are categorized as executive functions. Executive functions (also known as cognitive control and supervisory attentional system) are a set of cognitive processes - including attentional control, inhibitory control, working memory, and cognitive flexibility, as well as reasoning, problem solving, and planning - that are necessary for the cognitive control of behavior: selecting and successfully monitoring behaviors that facilitate the attainment of chosen goals. These functions have different components such as self-rule, working memory, and time estimation which evolve during growth process and together with getting older and help the person perform more complex and more difficult tasks. Damages to executive functions can have notable consequences in person's social, emotional, and cognitive functions. We can say that executive functions are defined as high level cognitive structures involved in adjustment of purpose-based behavior. Berg, Reijimer, Bresser, Kesseles, Kapplle, Biessels (2010) studies cognitive functions of patients with type 2 diabetes during 4 years and, utilizing WCST, showed a significant difference between functions of patients with diabetes and healthy people. On the other hand, Awad, Gangnon, and Messier (2004) found out about a relation between glucose changes in diabetes patients and their cognitive functions. Although the relation between effects of type 2 diabetes and cognitive functions is not clear, however, studies show deficiencies in planning, coordination, continuity, and selfadjustment in patients with type 2 diabetes (Yeung, Fischer, Dixon, 2009 and Qiu, Price, Hibberd 2006). Perhaps one of 
the most powerful observations for deficiencies of executive functions is the study by Yeung et al (2009) which is conducted on 465 people of whom 41 had type 2 diabetes. They found that the score of patients with type 2 diabetes in preventive functions, alternativeness, and reply is $12 \%$ to $14 \%$ less than healthy subjects. The effect was observed even after dividing the sample in the two young-old (53-70) and old-old (71-90) categories. They suggested that diabetes, compared to age, is a stronger predictor for deficiencies in executive functions with regards to the importance of executive functions in planning and alternativeness in patients with diabetes and lack of literature about the field in Iran, the researchers decided to conduct a study with the purpose of comparing executive functions in patients with diabetes and healthy people.

\section{Methodology}

\subsection{Population, samples and sampling}

As regards, Our intention was to identify changes in executive function in people with diabetes, The methodology of the study was causative-comparative and the population were patients with type 2 diabetes ranging from 20-50 years referring to Gabric association during Feb 2014 and May 2014. For sampling, by the available sampling method, 36 people referring for treatment were chosen based on their medical file and clinical interview. As for the normal group, with regards to age, sex, education and marital status, ordinary people were chosen amongst patients' companions. Criteria for entering the group were 1) identification of type 2 diabetes 2) identification of type 2 diabetes, minimum one year before the study 3) ability to understand Persian language 4) intention and satisfaction together with awareness for contribution in the study and 5) referring to Gabric diabetes association and having a file with the doctor. Criteria for leaving the group were 1) getting type 1 diabetes 2) currently experiencing intensive impacts of diabetes 3) having other chronic diseases other than diabetes effects 5) retardedness or identification of other psychological disorders like temperamental and stress disorders before identifying diabetes 6) previously contributing to a study.

\subsection{Tool}

WCST: this test is one of the most known neurocognitive examining abstract reasoning, cognitive flexibility, doldrums, problem solving, concept formation, category change, ability to test hypothesis and error feedback, starting strategy, stopping the task and sustainable attention (Leen, Petrides, Petre, Worsley \& Dagher, 2007), was developed by Berg et al (2010) and revised by Hilton et al (1993) (quoted from Onner, Stickle, Love, Biranchini, Stanford, 2005). The test was first developed to assess abstract reasoning and thought amongst normal adults. However, researchers found that they could utilize it to study cognitive damages. WCST is sensitive to neural conditions like frontal lobe loss, and psychological disorders. Weak performance in WCST may result from damage to frontal lobe and executive functions (Leen et al, 2007). The study by Alexer et al (1992) on 30 psychic patients showed that the credit of score in WCST (repetition error) 0.29 and the credit of inside scores was 0.94 (Pooli et al, 2002). Lezack (1983) suggest the validity of the test for examining cognitive damages following brain damage to be 0.86 . The credibility of the test based on Spirine, Stravess, Hesie (1991) equals to 0.83 . Ghadiri considers the credibility of the test to be 0.85 by the retesting method (Ghadiri, Jazayeri, Ashayeri, Ghazizade Tabatabayi, 2006).

\subsection{Method of Practice}

The study was conducted in two main steps. At first step, people referring to the center for treatment were introduced by the doctor and the psychologist of the center. Then, the medical file and assessment of standards, patients with the standards were chosen and interviewed individually. Besides, stating that the contributing to the study was voluntarily, the patients were assured that the results were fully confidential. Finally, patients who intended to contribute entered the second phase. At this step, first information regarding the disease records were gathered in an interview. Then, the demographic questionnaire was filled out and WCST categorization was done for individual patients.

\section{Findings}

Average and standard deviation of the patient sample were 34.29 and 4.03 respectively and of the healthy sample were 31.56 and 4.32. Using t-test, no significant difference between age averages was found which meant the group were unique regarding age. The table below shows results of t-test to compare executive functions between the two groups. 
Table 1: descriptive statistics and t-test results to compare executive functions of the patients group and the healthy group

\begin{tabular}{|l|c|c|c|c|c|c|}
\hline \multirow{2}{*}{ Test type } & \multicolumn{2}{|c|}{ Patient group } & \multicolumn{2}{c|}{ Healthy group } & \multirow{2}{*}{ t statistic } & \multirow{2}{*}{ Significance } \\
\cline { 2 - 7 } & Average & Standard deviation & Average & Standard deviation & & \\
\hline Completed categories & 3.12 & 1.73 & 5.41 & 0.97 & 7.176 & 0.0005 \\
\hline Doldrums error & 7.54 & 4.92 & 2.43 & 2.45 & 6.645 & 0.0005 \\
\hline
\end{tabular}

As we can see in the table above, there is a significant difference between the two groups, completed categories test $(\mathrm{t}=7$ / 176, $\mathrm{P}<0.001)$ and doldrums error $(\mathrm{t}=6 / 645, \mathrm{P}<0.001)$. Based on descriptive statistics, patients with type 2 diabetes compared to healthy subjects had weaker performance in WCST. This means that healthy subjects have a better performance in executive functions. The component probably shows their good ability and lack of disorder in concept creation. It also shows that patients with diabetes have problems in cognitive flexibility and utilize only one solution when facing problems.

\section{Discussion and Conclusions}

Results from the study showed that people with diabetes, compared to healthy counterparts, showed weaker performance in executive functions in each of WCST variables (category completion, doldrums error). The findings are in line with Kodl and Seaquist (2008), Qui et al (2006), Okereke et al (2008), Hull et al (2008) and Jurado and Rosselli (2007). Awad et al (2010), in a review study, showed that type 2 diabetes is more strongly correlated with memory deficiencies and executive functions.

The study showed that there is a significant difference between executive functions of the group, the healthy and the patient. In order to clarify findings, one can refer to Thabit, KyawTun, McDermott, Sreenan (2010). They examined the relation between duration of disease and executive functions and showed that they are directly related to resistance to insulin (one of diabetes symptoms), and that the more time it takes, the more is the risk of decrease in performance of executive functions.

It seems that that diabetes causes neurocognitive changes in the brain in two ways and affects executive functions. In a study conducted on 113 adults suffering from type 2 diabetes, 23\% showed disorders in cortex and 12\% showed disorders in the layer under the cortex. The study reported a significant relation between these disorders and the degree of deficiencies in attention, active memory and mind processing speed.

One of reasons diabetes causes deficiencies in executive functions is the role of Glycemic. In order to verify the relation, Munshi et al (2006) examined the correlation between blood sugar levels and active memory ( $r=-38)$. It seems that several factors together cause neurologic damages which is not yet clear.

The second way diabetes and executive functions are neurologically related, is the role of resistance to insulin. Although the process of relation between insulin and deficiencies of executive functions is not yet lucid, recent observations identify the role of insulin in lack of inhibition.

With regards to what was mentioned above, there are deficiencies in executive functions in diabetes patients as compared to healthy people, which have neurological themes, though not all the themes are clear. The findings can play an important role in training and self-management of people suffering from diabetes. Studies indicate the serious relation between diabetes and deficiencies in executive functions, and researchers should be trying to determine the relation. On the other hand, with regards to the findings, continuous examining of blood sugar levers seems necessary. The research faced limitations to which one should pay attention. First, the study is temporary, and in case of linear research, one can pay more attention to changes in executive functions during the time. Alternatively, we did not examine the effect of disease duration on executive functions which can be of importance. Our third limitation is the high range of executive functions, and our tool (WCST) examines only two of executive functions. Therefore, we recommend that the study be conducted utilizing extended tools and a higher range of executive functions be examined.

\section{Acknowledgement}

The study is a part of main author's dissertation submitted in partial fulfillment of the requirements for the degree of PhD in Psychology. 


\section{Resources}

Awad N, Gangnon M, Messier C: The relationship between impaired glucose tolerance, type 2 diabetes, and cognitive function.ClineNeuropsychological 2004, 26:1044-1080.

Berg EVD, Reijimer D, Bresser JD, Kesseles RPC, Kapplle LJ, Biessels GJ: A four year follow up study of cognitive functioning in patient with type 2 diabetes mellitus. Diabetologia 2010, 53:58-65.

Ghadiri, Fateme; Jazayeri, Alireza; Ashayeri, Hasan; and Ghazizade Tabatabayi, Mahmoud (2006) examining deficiencies in executive functions in schizophrenic patients, new trends in cognitive sciences, 8(3), 11-24.

Hull R, Martin RC, Beier ME, et al. Executive function in older adults: a structural equation modeling approach. Neuropsychology. 2008;22:508-522

Jurado MB, Rosselli M. The elusive nature of executive functions: a review of our current understanding. Neuropsychol Rev. 2007;17:213-233.

Kodl CT, Seaquist ER. Cognitive dysfunction and diabetes mellitus. Endocr Rev. 2008;29:494-511 SUE: 2

Leen, O., Petrides, M. Petre, V., Worsley, K., \& Dagher, A. (2007). Wisconsin card sorting revisited: Distinct neural circuits participating in different stages of the task identified by event-related functional magnetic resonance imaging. The Journal of Neuroscience, 21(19), 7733-7741.

Lezack B. Effect of Different Brain Lesions on Card Sorting. Archives of Neurology 1983; 9: 90-100.

Munshi M, Grande L, Hayes M, et al. Cognitive dysfunction is associated with poor diabetes control in older adults. Diabetes Care. 2006;29:1794-1799.

Norouzi, Azita; Ghafranipour, Fazlolla; Heidarnia, Alireza; Tahmasbi, Rahim (2010). Effective factors on regular physical exercise based on health improvement of women suffering from diabetes referring to Karaj diabetes association, Teb Jonoub Bimonthly, 13, 4151.

Okereke OI, Kang JH, Cook NR, et al. Type 2 diabetes mellitus and cognitive decline in two large cohorts of community-dwelling older adults. J Am Geriatr Soc. 2008;56:1028-1036.

Onner K. W, Stickle T. R, Love, J., Bianchini K. J, Stanford M. S. Latent structure of the Wisconsin Card Sorting Test: a confirmatory factor analytic study. Archives of Clinical Neuropsychology 2005; 20: 355-364.

Pooli A, Chan E, Tomassini A, Struglia F, Cavallaro R, Smeraldi E, Stratta P., The effect of Verbalization strategy on Wisconsin Card Sorting Test performance in schizophrenic patients receiving classical or atypical antipsychotics. BMC Psychiatry. 2002 Jan 26; 6:3.

Qiu WQ, Price LL, Hibberd P, et al. Executive dysfunction in homebound older people with diabetes mellitus. J A Geriatr Soc. 2006;54:496-501.

Spirine, A., Stravess, E.A., \& Hsieh, S. (1991). Shifting intentional set: Exploring the dynamic control of tasks. In C. Umiltà \& M. Moscovitch. Attention and performance XV: Conscious and nonconscious information processing (pp. 421- 452). Cambridge, MA: MIT Press Specka, R. \& Duncan, J. (2000). Neural mechanisms of selective visual attention. Annual Review of Neuroscience, $18,193-222$.

Thabit H,Kyaw Tun T,McDermott J,Sreenan S. (2010) Executive Function and Diabetes Mellitus - A Stone Left Unturned? Current Diabetes Reviews ;8,109-115 (7).

Unger J, McNeill TH, Moxley RT III, et al. Distribution of insulin receptor-like immunoreactivity in the rat forebrain. Neuroscience. 1989;31:143-157.

World Health Organization (2006). Prevalence of diabetes in the Who Eastern Mediterranean Region. Diabetes program; Available from: http:// www. who. int/ diabetes/facts/ world-figure/en/index2.html.

Yeung SE, Fischer AL, Dixon RA. Exploring effects of type 2 diabetes on cognitive functioning in older adults. Neuropsychology. 2009;23:1-9 